J. Lake Sci.(湖泊科学), 2020, 32(3): 654-664

DOI 10. 18307/2020. 0306

(c) 2020 by Journal of Lake Sciences

\title{
白洋淀表层沉积物中有机氯农药和全多氯联苯的分布特征及风险 评估
}

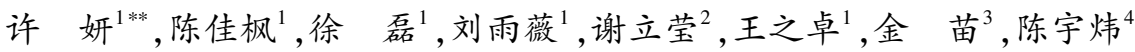 \\ (1: 东南大学土木工程学院,南京 210096) \\ ( 2 : 辽宁省兴城市环境保护监测站,兴城 125100$)$ \\ (3: 中国科学院南京地理与湖泊研究所, 南京 210008) \\ (4:南昌工程学院,南昌 330099)
}

摘 要: 为了解白洋淀表层沉积物中有机氯农药 ( OCPs) 和多氯联苯 (PCBs) 的污染情况, 采用改进的 GC- $\mu \mathrm{ECD}$ 方法对 白洋淀 11 处沉积物进行了 20 种 OCPs 和全部 209 种 PCB 单体的定量检测和分析. 结果显示: 白洋淀 11 个沉积物样品共 检出 10 种 OCPs 和 24 种 PCBs, $\sum O C P$ s 和 $\sum$ PCBs 的含量范围分别为 $1.22 \sim 52.45 \mathrm{ng} / \mathrm{g}(\mathrm{DW})$ 和 $\mathrm{nd} \sim 37.61 \mathrm{ng} / \mathrm{g}$, 在国内处 于中等水平; $\mathrm{OCPs}$ 组成中以 $\mathrm{HCHs}$ 和 Dieldrin (狄氏剂) 为主, 分别占到 $\Sigma O C P s$ 的 $39.9 \%$ 和 $31.5 \%$, 其中 7 个采样点的 $\mathrm{HCHs}$ 以林丹输人为主, 4 个采样点以工业六六六污染为主. DDTs 检出率较低, 来源主要为历史残留; 检出的 PCB 单体以 低氯联苯为主, 其中一氯、二氯和三氯联苯占 $\sum$ PCBs 的 $64.73 \%$; 采用沉积物质量标准法进行生态风险评估, 结果表明白 洋淀地区沉积物中 $\mathrm{p}, \mathrm{p}^{\prime}-\mathrm{DDD}$ 和 $\Sigma$ PCBs 生态风险较低, Dieldrin 生态风险尚需关注, $\gamma$-HCH 生态风险较高, 不容忽视.

关键词: 白洋淀;沉积物;多氯联苯; 有机氯农药; 风险评估

\section{Distribution and risk assessment of organochlorine pesticides and polychlorinated biphen- yls in surficial sediments from Lake Baiyangdian *}

XU Yan ${ }^{1 * *}$, CHEN Jiafeng ${ }^{1}$, XU Lei $^{1}$, LIU Yuwei $^{1}$, XIE Liying $^{2}$, WANG Zhizhuo $^{1}$, JIN Miao $^{3}$ \& CHEN Yuwei $^{4}$

(1: School of Civil Engineering, Southeast University, Nanjing 210096, P.R.China)

(2: Environmental Protection Monitoring Station of Xingcheng City, Liaoning Province, Xingcheng 125100, P.R.China)

(3: Nanjing Institute of Geography and Limnology, Chinese Academy of Sciences, Nanjing 210008, P.R.China)

(4: Nanchang Institute of Technology, Nanchang 330099, P.R.China)

Abstract: To better understand the contamination of organochlorine pesticides (OCPs) and polychlorinated biphenyls (PCBs) in sediments from Lake Baiyangdian, 20 OCPs and all 209 PCB congeners were measured in 11 surficial sediment samples collected from different locations of Lake Baiyangdian. Moreover, the risks posed by OCPs and PCBs were assessed based on sediment quality guidelines. The results show that 10 OCPs and 24 PCB congeners were detectable. The total OCPs concentrations ranged from 1.22 to $52.45 \mathrm{ng} / \mathrm{g}(\mathrm{DW})$ (dry weight), while the total PCBs levels were from below detection limit to $37.61 \mathrm{ng} / \mathrm{g}$. These results indicate a moderate pollution level within China. HCHs and Dieldrin were predominant in all OCPs, which accounted for 39.9\% and $31.5 \%$ of total OCPs, respectively. Seven sampling sites of Lake Baiyangdian were contaminated by Lindane $(\gamma$-HCH $)$ and the other 4 sampling sites were mainly contaminated by industrial HCHs. Moreover, new HCHs inputs might exist in some locations. DDTs levels were relatively low, which suggests that DDTs in Lake Baiyangdian might be the historical residues. PCBs detected in these sediment samples were dominated by low chlorinated PCBs congeners. Mono-, di-and tri-chlorinated biphenyls accounted for

* 2019-05-06 收稿;2019-11-13 收修改稿.

国家自然科学基金项目 (41671468,41301546,51408119)、江苏省自然科学基金项目 (BK20171356) 和江苏省青蓝 工程和污染控制与资源化国家重点实验室开放基金项目 (PCRRF16018) 联合资助.

** 通信作者;E-mail:xuxucalmm@ seu.edu.cn. 
$64.73 \%$ of the total PCBs. However, it is noted that some new organochlorine pesticide pollution of HCHs may be present in some areas. Ecological risk assessment based on sediment quality guidelines revealed that the risks posed by $\mathrm{p}, \mathrm{p}^{\prime}$-DDD and $\sum$ PCBs were relatively lower, while the risk associated with Dieldrin was of concern. Notably, $\gamma$-HCH posed apparent adverse effects on the local ecological system.

Keywords: Lake Baiyangdian; sediments; polychlorinated biphenyls; organochlorine pesticides; risk assessment

有机氯农药 (Organochlorine pesticide, OCPs) 和多氯联苯 (Polychlorinated biphenyls, PCBs) 是常见的两类 有机氯污染物, 具有 “三致” 毒性, 近来研究更表明其会干扰生物内分泌系统、影响儿童智力发育 ${ }^{[1]}$. 由于 OCPs 和 PCBs 分别在农业生产 (杀虫) 和工业应用 (油漆、变压器油、电容器介电流体等) 上表现卓越, 曾在 全球范围内被大量生产和使用. 尽管 1970s 后, OCPs 和 PCBs 的生产和使用受到了严格的限制, 但由于其理 化性质稳定, 至今仍是备受关注的全球性污染问题. 1979 年 PCBs 被美国环境保护署( USEPA) 列人优先检 测物黑名单,全美禁用; 2001 年斯德哥尔摩公约正式将 PCBs、Aldrin、Dieldrin、Endrin、DDT、HCB、Heptachlor、 Chordane 列为 8 种 (共 12 种) 持久性有机污染物; 2013 年, 在美国颁布的国家有害物质优先清单中, PCBs 位 列第 5. 中国在 $1980 \mathrm{~s}$ 前曾生产过大量的 OCPs, 其中以 $\operatorname{HCHs}$ ( 450 万吨) 和 DDTs ( 27 万吨) 为主 ${ }^{[2-3]}$. 同时, 统计显示, 我国曾生产过近万吨的 $\mathrm{PCBs}{ }^{[4]}$, 并进口过大量的电力电容设备 (含 PCBs) ${ }^{[3]}$, 因历史局限性, 这 些 PCBs 存在较高泄露风险 ${ }^{[5]}$. 调查发现, OCPs 和 PCBs 有机氯污染物在国内的空气、土壤、河流湖泊以及沉 积物中均有检出 ${ }^{[6-9]}$. 白洋淀 $\left(38^{\circ} 43^{\prime} \sim 39^{\circ} 02^{\prime} \mathrm{N}, 115^{\circ} 38^{\prime} \sim 116^{\circ} 07^{\prime} \mathrm{E}\right)$, 水域面积约为 $366 \mathrm{~km}^{2}$, 平均水深 $2 \sim$ $3 \mathrm{~m}$, 作为华北地区最大的浅水型湖泊,被誉为“华北之肾”. 2017 年雄安新区设立后,白洋淀也成为新区的 重要水源地之一. 有机氯污染物因为疏水性,河流湖泊沉积物是其主要的“汇”. 2010-2014 年, 虽然白洋淀 有机氯污染物的调查发现了 HCHs、DDTs 和 PCBs 等污染物, 存在不利生物风险 (抚河地区), 但总体上仍较 为有限 ${ }^{[10-14]}$. 本研究于雄安新区设立之初的 2017 年进行, 以期为新区建设的生态影响提供科学依据. 此外, 受限于仪器分析方法, 白洋淀地区 PCBs 的研究大多用几种到几十种 PCBs 单体来代表 PCBs 总量, 而徐磊 等 ${ }^{[15]}$ 发现有限的 PCBs 单体检测会导致对环境中 PCBs 污染程度的低估. 本研究希望通过对全部 209 种 PCBs 检测获得更加全面的数据.

本文以白洋淀 11 个主要人类活动区域的表层沉积物为研究对象, 对 20 种 OCPs 和 209 种 PCBs 含量进 行检测, 并探讨其来源, 同时评估其生态风险, 为白洋淀水环境中有机氯污染防治和雄安新区生态环境综合 整治提供科学依据和数据支持.

\section{1 材料与方法}

\section{1 样品采集}

在白洋淀水域内选取主要围绕人类活动地区重要水道和面积较大的淀, 于 2017 年 5 月利用手持式 GPS 定位系统, 选定 11 处采样点并标号 BY1 BY11, 依次代表苲淀、荷花淀、杨家淀、光淀、鹗鹩岛、李郎村、烧车 淀河道、烧车淀中心、郭里口、大观园和烧车淀湿地. 各个采样点水深 $0.43 \sim 0.97 \mathrm{~m}$, 水温 $18.81 \sim 20.86^{\circ} \mathrm{C}$. 使 用抓斗式采泥器在每个采样点采集 $1 \sim 2 \mathrm{~L}$ 的 $0 \sim 10 \mathrm{~cm}$ 表层沉积物样品, 去除多余的杂质、动植物残体等, 水 封于棕色嗍玻璃瓶中, 并置于低温 $\left(4^{\circ} \mathrm{C}\right)$ 条件下备用. 11 处采样点的具体位置、采样环境及地理分布如图 1 所示.

\section{2 仪器与试剂}

主要仪器: Agilent 7890A 气相色谱一电子捕获检测器 (GC- $\mu$ ECD, 美国安捷伦科技有限公司); Eyela N$1200 B$ 旋转蒸发仪 (东京理化器械株式会); Eyela MGS-220 氮吹仪 (东京理化器械株式会);ZX3 浴旋仪 (美 国赛默飞世尔科技).

主要试剂和药品: 美国天地品牌, 色谱纯, 正已烷 (n-Hexane 95\%)、丙酮 ( Acetone)、二氯甲烷 (Dichloromethane)、异丙醇 ( Isopropanol) ; 美国赛默飞世尔科技 60 100 目弗罗里土(Florisil); 美国西格玛奥德里奇 品牌, 分析纯, 无水硫酸钠 $\left(\mathrm{Na}_{2} \mathrm{SO}_{4}\right)$ 、氯化钠 $(\mathrm{NaCl})$ 、亚硫酸钠 $\left(\mathrm{Na}_{2} \mathrm{SO}_{3}\right)$ 、四丁基硫酸氢铵 $(\mathrm{TBA})$ 等; 美国 AccuStandard 公司的多氯联苯单体及混合标准样品; OCPs 标准物质和内标化合物: 滴滴涕及其代谢产物系 列; HCHs 系列, 狄氏剂(Dieldrin)、异狄氏剂(Endrin)、艾氏剂(Aldrin)、硫丹 I (Endosulfan I)、硫丹 II（En- 


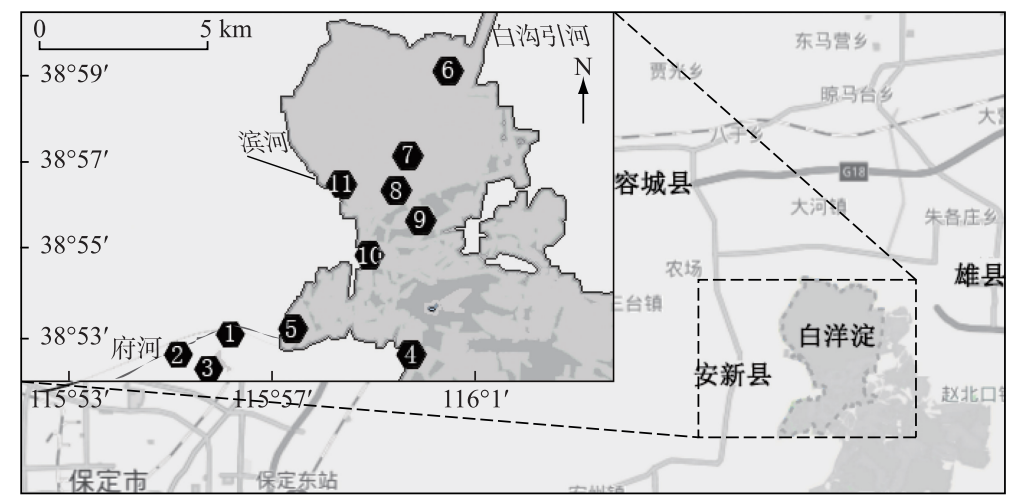

图 1 白洋淀表层沉积物采样点分布

Fig. 1 Sampling sites of surficial sediments in Lake Baiyangdian

dosulfan II)、硫丹硫酸酯(Endosulfan sulfate)、十氯酮 (Kepone)、甲氧滴滴涕 (Methoxylor)、七氯( Heptachlor)、 七氯环氧 (Heptachlor epoxide)、六氯苯 (Hexachloro- benzene, HCB) 、灭蚁灵 (Mirex)、氯丹(Chlordane, 含顺反 式) 共 20 种 OCPs 标准物质和内标化合物五氯硝基苯 (PCNB) 以及回收指示物十氯联苯 (PCB209) 均购自 美国 Accustandard 公司.

\section{3 样品处理}

样品有机氯污染物的萃取采用加速溶剂萃取法 (ASE) ( 美国环境保护署《加压液体萃取 (PFE)》( EPA Method 3545A)). 沉积物样品自然风干后充分研磨并过 60 目不锈钢篮篮分; 将 $5.0 \mathrm{~g}$ 沉积物样品和 $5.0 \mathrm{~g}$ 预 处理过 (马弗炉 $450^{\circ} \mathrm{C}, 4 \mathrm{~h}$ ) 的石英砂加人放有纤维素滤膜的不锈钢萃取池中 ( $34 \mathrm{~mL}$ ), 充分混合均匀. 于萃 取池中; 以正已烷/丙酮 (1:1) 混合液进行加压萃取. 设定的萃取程序包括: 预热 $5 \mathrm{~min}$, 静态萃取 $5 \mathrm{~min}$, 萃取 压力 $10.3 \mathrm{MPa}$, 萃取温度 $100^{\circ} \mathrm{C}$, 冲洗体积 $60 \%$, 循环萃取 2 次. 萃取完成后, 转移萃取并液氮吹浓缩至 $1 \mathrm{~mL}$.

萃取液的净化采用美国环境保护署《硫的净化》( EPA Method 3660B) 和《硅酸镁载体柱净化》(EPA Method 3620) 分别于萃取液中加人 $1 \mathrm{~mL}$ 四丁基铵-亚硫酸钠 (TBA) 溶液和 $2 \mathrm{~mL}$ 异丙醇, 振荡至底部有晶体 出现. 加人 $4 \mathrm{~mL}$ 超纯水充分摇匀至晶体溶解, 静置分层, 转移有机层 (上层) 溶液并氮吹浓缩至 $1 \mathrm{~mL}$. 于直 径为 $10 \mathrm{~mm}$ 的 $30 \mathrm{~cm}$ 玻璃层析柱中自下而上填充 $8.0 \mathrm{~g}$ 活化的弗罗里硅土和 $3.0 \mathrm{~g}$ 预处理过的无水硫酸钠 (马弗炉 $450^{\circ} \mathrm{C}, 6 \mathrm{~h}$ ) ; 分别用 $90 \mathrm{~mL}$ 正已烷和 $90 \mathrm{~mL}$ 正已烷/二氯甲烷 (4:1) 混合液梯度洗脱. 洗脱液旋蒸浓 缩、氮吹后定容至 $200 \mu \mathrm{L}$ 待进样.

\section{4 气相色谱分析}

采用配置 ${ }^{63} \mathrm{Ni}$ 微电子捕获检测器的气相色谱仪 (GC- $\mu$ ECD) (Agilent 7890A) 对 OCPs 和 PCBs 进行定量

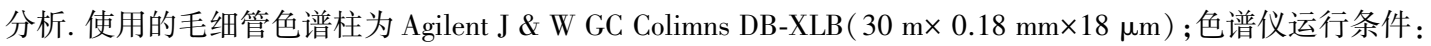
进样口温度 $275^{\circ} \mathrm{C}$, 检测器温度 $300^{\circ} \mathrm{C}$, 载气为高纯氦气 $(99.999 \%)$. 升温程序: 初始柱温 $50^{\circ} \mathrm{C}$, 保持 $1 \mathrm{~min}$, 然 后以 $12^{\circ} \mathrm{C} / \mathrm{min}$ 升至 $150^{\circ} \mathrm{C}$, 接着以 $0.4^{\circ} \mathrm{C} / \mathrm{min}$ 升至 $220^{\circ} \mathrm{C}$, 最后以 $2^{\circ} \mathrm{C} / \mathrm{min}$ 升至 $260^{\circ} \mathrm{C}$. 进样量为 $1 \mu \mathrm{L}$, 采用 不分流进样.

\section{5 质量控制}

在上述色谱条件下, 利用 20 种 OCPs 和 209 种 PCBs 的标样获得各物质色谱峰保留时间, 据此对实际样 品中的 OCPs 和 PCBs 进行定性. 采用多点校正曲线法对样品进行外标定量分析, 方法检测限 $<1 \mathrm{ng} / \mathrm{mL}$. 采 用方法空白、平行样和加标回收对实验进行 QA $/ Q C$ 控制. 过程空白样中均无 OCPs 和 PCBs 检出; 平行样相 对偏差 $<5 \%$, OCPs 和 PCBs 的平均加标回收率为 $85.3 \% \sim 105.5 \%$ 和 78.2\% 102.6\% ( 详细见附录 I 、 II ).

\section{6 风险评估方法}

鉴于国内无相应 OCPs 和 PCBs 沉积物质量标准, 本研究参考国外沉积物质量标准法 ( sediment quality guidelines, SQGs) 中使用较多的 Long 等 ${ }^{[16]}$ 提出的生物毒性影响标准中的 $E R L($ effects range low, 低于该值毒 
性风险小于 $25 \%$ )、 $E R M($ effects range median, 高于该值毒性风险大于 $75 \%$ ) 值和加拿大魁北克省的标准中 的 TEL (the threshold effect level, 低于该值几乎不会引起生物负效应)、PEL (the probable effect level, 高于该 值将有较大可能产生不利反应) 值对白洋淀地区沉积物中的 OCPs 和 PCBs 进行风险评估. 同时, 根据检出 的 OCPs 和 PCBs, $m E R M q$ (mean $E R M$ quotient) 值将被用于污染物的综合风险评估,计算公式为:

$$
{ }_{m E R M}=\left(\sum C_{x} / E R M_{x}\right) / n
$$

式中, $C_{x}$ 指污染物 $x$ 的检出浓度, $E R M_{x}$ 指污染物 $x$ 对应的 $E R M$ 值或 PEL 值(出于风险评估保守性, 部分 $E R M$ 值用 $P E L$ 值代替) , $n$ 表示参与综合风险评估的污染物的数量. 归类为: $m E R M q<0.1$, 风险最小, 毒性概 率仅为 $9 \% ; 0.1 \leqslant m E R M q<0.5$, 低风险, 毒性概率为 $21 \% ; 0.5 \leqslant m E R M q<1.5$, 中风险, 毒性概率为 $49 \%$; $m E R M q \geqslant 1.5$, 高风险, 毒性概率为 $76 \%$.

\section{2 结果与讨论}

\section{1 白洋淀表层沉积物中 OCPs 分布特征及分析}

白洋淀 11 处表层沉积物的 $\Sigma$ OCPs 含量在 1.22 $52.45 \mathrm{ng} / \mathrm{g}$ 之间, 均值为 $16.12 \mathrm{ng} / \mathrm{g}$, 与 $\mathrm{Hu}$ 等 ${ }^{[17]}$ 的研 究结果相近. 11 处表层沉积物采样点中, BY5 (鹗學 岛)、BY9(郭里口) 和 BY11 ( 烧车淀湿地) 处的 $\Sigma O C P s$ 含量相对较高, BY6(李郎村) 和 BY10(大观园) 处的 EOCPs 含量相对较低 (图 2). 考虑到駕䔄岛, 郭里口 和烧车淀湿地均为白洋淀的重要风景区, 这 3 处地区 较高的 $\Sigma O C P s$ 含量可能与频繁的人类活动有关, 而 烧车淀湿地在三者中最高的 $\Sigma$ OCPs 含量这一结果则 还应考虑到湿地的生态截留作用 ${ }^{[18]}$. 附录 III 和表 1 中这 3 处区域较高含量的 Aldrin、Endosulfan sulfate、 Endrin ketone 检出和相应较低的检出率印证了这 3 处地区较其他研究区域农药应用广泛的说法.

白洋淀表层沉积物中 OCPs 分布情况如表 1 所 示, 所检测的 20 种 OCPs 在所有采样点中共检出 10 种, 分别为 $\alpha-\mathrm{HCH} 、 \beta-\mathrm{HCH} 、 \gamma-\mathrm{HCH} 、 \delta-\mathrm{HCH} 、$ Aldrin 、 Dieldrin 、p-p'-DDD、Endosulfan sulfate、Endrin ketone 和 methoxychlor. 检出率较大 $(>50 \%)$ 的 OCPs 共有 3 种, 其中 $\alpha-\mathrm{HCH}$ 在所有采样点均有检出, 含量范围为 $0.41 \sim 4.57 \mathrm{ng} / \mathrm{g}$, 其次为 Dieldrin, 检出率达 $82 \%$, 含 量范围为 $\mathrm{nd} \sim 19.83 \mathrm{ng} / \mathrm{g}, \boldsymbol{\gamma}-\mathrm{HCH}$ 的检出率和含量范 围分别为 $64 \%$ 和 nd $20.77 \mathrm{ng} / \mathrm{g}$, 而其余 7 种检出的 OCPs 检出率均较低, 仅在白洋淀个别地区有检出. OCPs 分布情况显示 HCHs 和 Dieldrin 类农药曾在白 洋淀地区使用广泛, 这与国内湖泊的 OCPs 含量分布 相似 ${ }^{[24,27,33]}$, 而其余类型的农药使用较少且分布不 均匀, 只在局部地区使用.

国内不同地区水体沉积物中的 $\Sigma O C P s$ 含量见表 2. 与国内其他研究区域相比, 白洋淀地区的 $\Sigma O C P s$ 含 量高于松花江流域、千岛湖和长江中下游, 接近于韩江流域、太湖梅梁湾和博斯腾湖等, 较其他水体沉积物 中的 $\Sigma O C P$ s 含量低. 总体来说, 白洋淀地区表层沉积物中的 $\Sigma O C P s$ 含量在我国处于中等水平, 与 2010 年白 洋淀调查所显示的 $\Sigma O C P s$ 平均含量无明显差异.

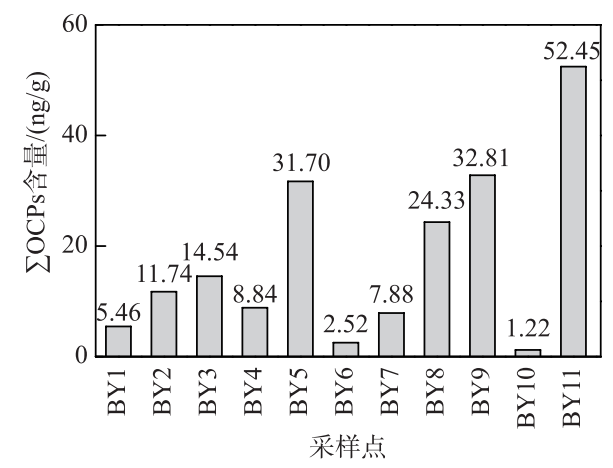

图 2 白洋淀表层沉积物中 $\sum O C P$ s 含量

Fig. 2 Content of total OCPs in surficial sediments from Lake Baiyangdian

表 1 白洋淀表层沉积物中 OCPs 分布情况 *

Tab.1 Distribution of OCPs in surficial sediments from Lake Baiyangdian

\begin{tabular}{ccccc}
\hline OCPs & 检出率/\% & $\begin{array}{c}\text { 范围/ } \\
(\mathrm{ng} / \mathrm{g})\end{array}$ & $\begin{array}{c}\text { 均值/ 标准差/ } \\
(\mathrm{ng} / \mathrm{g})\end{array}$ \\
$\begin{array}{ccccc}(\mathrm{ng} / \mathrm{g}) \\
\alpha \text {-HCH }\end{array}$ & 100 & $0.41 \sim 4.57$ & 1.26 & 1.3 \\
$\beta$-HCH & 36 & $\mathrm{nd} \sim 3.09$ & 0.92 & 1.3 \\
$\gamma$-HCH & 64 & $\mathrm{nd} \sim 20.77$ & 4.65 & 6.1 \\
$\delta$-HCH & 9 & $\mathrm{nd} \sim 2.03$ & 0.18 & 0.6 \\
Aldrin & 27 & $\mathrm{nd} \sim 5.71$ & 1.88 & 4.2 \\
Dieldrin & 82 & $\mathrm{nd} \sim 19.83$ & 5.54 & 5.4 \\
p,p'-DDD & 18 & $\mathrm{nd} \sim 2.66$ & 0.45 & 1.0 \\
Endosulfan sulfate & 9 & $\mathrm{nd} \sim 5.46$ & 0.50 & 1.6 \\
Endrin ketone & 9 & $\mathrm{nd} \sim 3.41$ & 0.31 & 1.0 \\
methoxychlor & 18 & $\mathrm{nd} \sim 11.30$ & 1.90 & 4.2 \\
\hline
\end{tabular}

* 样品数 (11 处采样点). 
表 2 国内不同地区表层沉积物中 OCPs 含量比较

Tab.2 Comparison of OCPs content in surficial sediments from different domestic areas

\begin{tabular}{|c|c|c|c|c|}
\hline 采样地区 & 年份 & $\Sigma O C P s /(n g / g)$ & 平均值 $/(\mathrm{ng} / \mathrm{g})$ & 参考文献 \\
\hline 长江中下游 & 2019 & $0.038 \sim 11.36$ & - & {$[19]$} \\
\hline 韩江流域(广东) & 2018 & $2.97 \sim 40.54$ & 10.57 & {$[20]$} \\
\hline 沙英江( 河南) & 2018 & $12.3 \sim 77.5$ & 44.6 & {$[21]$} \\
\hline 会仙湿地湖泊(广西) & 2018 & $68.7 \sim 305$ & 137 & {$[22]$} \\
\hline 白洋淀 (河北) & 2017 & $1.22-52.45$ & 16.12 & 本研究 \\
\hline 博斯腾湖 (新疆) & 2017 & $6.9 \sim 16.7$ & 11.1 & {$[23]$} \\
\hline 鄱阳湖周溪湾( 江西) & 2016 & $40.4 \sim 174.1$ & 104.1 & {$[24]$} \\
\hline 巢湖(安徽) & 2016 & $11 \sim 26.3$ & 18.8 & {$[25]$} \\
\hline 海河上游(京津) & 2015 & $2.75 \sim 139$ & 40.1 & {$[26]$} \\
\hline 太湖梅梁湾 ( 江苏) & 2015 & $2.68 \sim 31.34$ & 19.43 & {$[27]$} \\
\hline 千岛湖 (浙江) & 2014 & $0.43 \sim 12.70$ & - & {$[28]$} \\
\hline 白洋淀 (2010 年) & 2010 & $12.1 \sim 15.8$ & 14.20 & {$[10]$} \\
\hline 松花江( 黑龙江) & 2008 & $0.7 \sim 6.07$ & 2.87 & {$[29]$} \\
\hline 南四湖 (山东) & 2007 & $1.64 \sim 17.9$ & - & {$[30]$} \\
\hline 河汊湖 (湖北) & 2007 & $37.16 \sim 154.50$ & - & {$[31]$} \\
\hline 红枫湖 (贵州) & 2006 & $11.8 \sim 48.6$ & 22.8 & {$[32]$} \\
\hline
\end{tabular}

DDT 及其降解产物 DDD、DDE 的相对含量和 HCHs 各异构体 ( $\alpha-\mathrm{HCH}, \beta-\mathrm{HCH}, \gamma-\mathrm{HCH}, \delta-\mathrm{HCH}$ ) 的比例 常被用于研究环境中 OCPs 的代谢情况和污染源的分析. 自然环境中的 DDT 有两种归宿 ${ }^{[34]}$, 视好氧环境和 厌氧环境的不同,DDT 可分别被相应的好氧或厌氧微生物降解为 DDE 和 DDD, 一般以 DDE 与 DDD 之和相 对 DDT 的含量 $[m(\mathrm{DDE})+m$ ( DDD ) $] / m$ ( DDTs) 作为判断有无新污染源进人水体的指标 $(>0.5)$. 进一步分 析表 1 数据发现, p, $\mathrm{p}^{\prime}$-DDT 和 $\mathrm{p}, \mathrm{p}^{\prime}$-DDE 均未被检出, 仅有少量 $\mathrm{p}, \mathrm{p}^{\prime}$-DDD 在 BY3 (杨家淀) 和 BY11 (烧车淀 湿地) 处发现, 表明白洋淀底泥中的 DDTs 污染来源为历史残留, 无新 DDT 污染源输人, 且底泥中 DDT 降解 主要为厌氧降解.

$\mathrm{HCHs}$ 与 Dieldrin 是本研究中的主要污染物, 环境中的 $\mathrm{HCHs}$ 主要来源于工业生产的六六六和林丹产 品,一般工业六六六中 $\alpha$-HCH 比重为 $60 \% \sim 70 \%, \beta$-HCH 比重为 5\% 12\%, $\gamma$-HCH 比重为 $10 \% \sim 15 \%$, $\delta$ - $\mathrm{HCH}$ 比重为 6\% 10\%, 而林丹中 99\%为 $\gamma-\mathrm{HCH}$. 故研究中常用 $\alpha-\mathrm{HCH}$ 与 $\gamma-\mathrm{HCH}$ 的比值 $m(\alpha-\mathrm{HCH}) / m$ $(\gamma-\mathrm{HCH})$ 来估计污染点处 $\mathrm{HCHs}$ 的来源 ${ }^{[35]}, m(\alpha-\mathrm{HCH}) / m(\gamma-\mathrm{HCH})$ 在 4 7 之间认为是工业六六六残留的 结果, 而当 $m(\alpha-\mathrm{HCH}) / m(\gamma-\mathrm{HCH})$ 小于 1 趋近 0 时则认为该处污染源自林丹的输人. 同时, $\mathrm{HCHs}$ 的 4 种单

$\square \alpha-\mathrm{HCH} \quad \square \gamma-\mathrm{HCH} \square \beta-\mathrm{HCH} \quad \square \delta-\mathrm{HCH}$

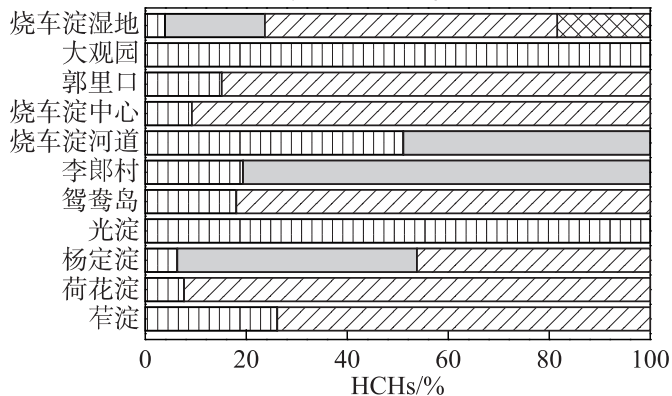

图 3 白洋淀表层沉积物中 HCHs 异构体组成

Fig.3 Percentage composition of $\mathrm{HCHs}$ in surficial sediments from Lake Baiyangdian 体中 $\beta$-HCH 的物理化学性质最为稳定,其他 3 种环 境中的 $\mathrm{HCH}$ 单体会随时间向 $\beta-\mathrm{HCH}$ 转化, 较高的 $\beta$ $\mathrm{HCH}$ 含量可表明环境中无新的 HCHs 污染源进入.

从白洋淀 11 处采样点沉积物中各 $\mathrm{HCH}$ 异构体 比例分布 (图 3) 可知, 苲淀、荷花淀、杨家淀、駕春岛、 烧车淀中心、郭里口和烧车淀湿地处的 $m(\alpha-\mathrm{HCH}) /$ $m(\gamma-\mathrm{HCH})$ 范围在 $0.07 \sim 0.35$ 之间, 均趋近于 0 , 说明 该 7 处地区的 $\mathrm{HCH}$ s 输人以林丹为主. 进一步分析该 7 处地区, 杨家淀和烧车淀湿地两处的 $\beta-\mathrm{HCH}$ 含量 ( $19.8 \%$ 和 $47.5 \%)$ 均较工业产品中的 $\beta$-HCH 含量 $(5 \% \sim 12 \%)$ 高, 表明这两处区域的 HCHs 污染主要为 历史残留, 而其余 5 处地区均无 $\beta$-HCH 检出, 对比 2008 年 Dai 等 ${ }^{[36]}$ 得到的白洋淀底泥中较高 $\beta$ - HCH 的 调查结果, 白洋淀地区可能存在新的林丹的使用, 在 
高秋生等 ${ }^{[33]}$ 最近对白洋淀底泥的研究中亦有类似的发现. 其余 4 处采样点 (光淀、李朗村、烧车淀河道和大 观园地区) 沉积物中均无 $\gamma-\mathrm{HCH}$ 检出且 HCHs 异构体以 $\alpha-\mathrm{HCH}$ 为主,表明这 4 处地区的 HCHs 主要来源于 工业六六六的污染. 四处采样点中李朗村和烧车淀河道检 出高比例的 $\beta$-HCH 含量 ( $80.6 \%$ 和 $48.8 \%$ ), 表明该地区无 新的 HCHs 污染, 而光淀和大观园地区则无 $\beta-H C H$ 检出, 表明可能存在新的工业六六六输人. 综上所述,白洋淀 11 处采样点有 7 处受林丹污染, 4 处受工业六六六污染,其中 有 7 处采样点附近区域可能存在新的 $\mathrm{HCHs}$ 类农药的 使用.

在污染源分析中,主成分分析 (PCA) 方法应用较为广 泛. 利用 Origin 9 软件对白洋淀 11 处采样点检出的 10 种 OCPs 进行主成分分析,前两个主成分 PC1 和 PC2 的方差 解释量分别为 $45.55 \%$ 和 $21.11 \%$, 总方差解释量达到 $66.66 \%$, 结果如图 4 所示. 由图 4 可知, BY5 (鹗鹌岛) 和 BY8 (烧车淀中心) 污染情况相似; BY1 (窄淀)、BY2(荷花 淀)、BY4(光淀) 和 BY10 (大观园) 污染情况相似; BY6(李 朗村) 和 BY7 (烧车淀河道) 污染情况相似,这代表这些区 域间可能存在相同的污染源或具有相互扩散的可能,而 BY3 (杨家淀)、BY9 (郭里口) 和 BY11 (烧车淀湿地) 与其 他淀的污染特征有较大差异. 从总体上分析,各点位在 PCA 图上没有显著的聚类归一现象,这可能说明白洋淀 OCPs 农药种类使用并不统一.

\section{2 白洋淀表层沉积物中 PCBs 分布特征及源分析}

209 种 PCB 单体在白洋淀 11 处采样点表层沉积物中 共检出 24 种, 总 PCBs 含量在 $\mathrm{nd} \sim 37.61 \mathrm{ng} / \mathrm{g}$ 之间, 平均 含量为 $13.45 \mathrm{ng} / \mathrm{g}$. 各采样点处 PCBs 种类和总含量见图 5 和附录 $I V$, 其中 BY11 (烧车淀湿地) 处总 PCBs 含量最 高, 为 $37.61 \mathrm{ng} / \mathrm{g}$, 该地检出的 PCB 单体及同系物种类也 最多, PCBs 污染严重; BY3 (杨家淀) 和 BY5 (鹗葟岛) 位 居第 $2 、 3$, 分别为 29.27 和 $24.30 \mathrm{ng} / \mathrm{g}$, 其余地区总 $P C B s$ 含量较前三者低,而BY6(李朗村) 低于检测限.

多氯联苯同系物组成常用来分析环境中 PCBs 的分 布、来源和归宿. 本研究白洋淀地区表层沉积物中 PCBs 以低氯联苯为主,一氯联苯最多, 占总量的 $31.16 \%$, 二氯 联苯和三氯联苯其次, 分别占总量的 $17.60 \%$ 和 $15.97 \%$, 四 氯联苯含量占总量的 $11.49 \%$. 高氯联苯中六氯联苯居多, 占总量的 $12.55 \%$, 五氯联苯相对含量仅为总量的 5.3\%, 七氯联苯为 $4.6 \%$, 其余高氯联苯含量可忽略不计 (图 6). 中国在 1980s 前生产的 PCBs 商品多以三氯联苯和五氯联 苯为主, 其中三氯联苯主要用于电容器, 而五氯联苯多用 于油漆. Dai 等 ${ }^{[36]}$ 在 2008 年对白洋淀地区沉积物 PCBs 的 调查中发现, 白洋淀沉积物中 PCBs 以高氯联苯为主 (五 氯联苯和六氯联苯占总量的 $65 \%$ ), 并推测船底油漆是白 洋淀地区主要的 PCBs 污染来源. 对比 10 年前白洋淀沉积

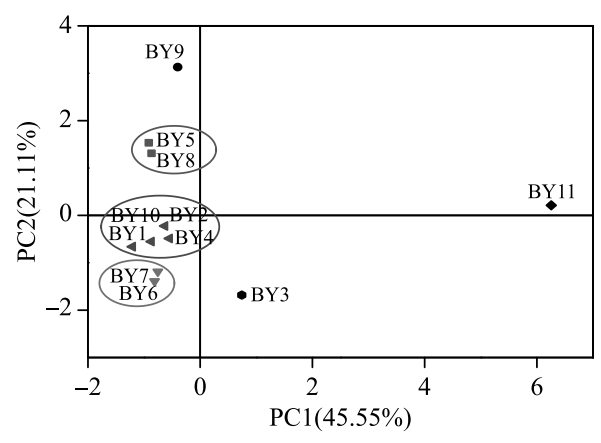

图 4 白洋淀表层沉积物中 OCPs 主成分分析

Fig.4 Principal component analysis of OCPs in surficial sediments from Lake Baiyangdian

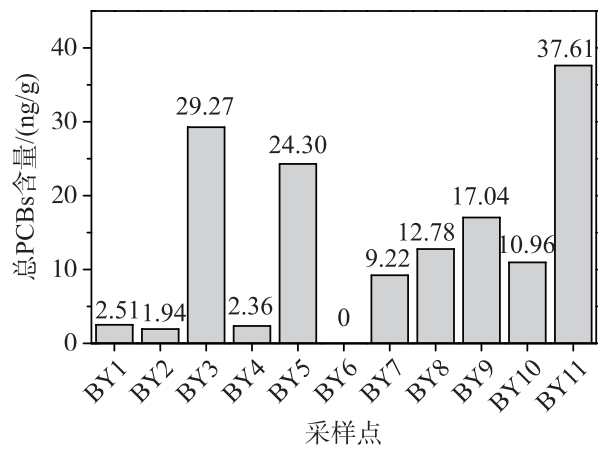

图 5 白洋淀表层沉积物中检出的 PCBs 单体及含量分布

Fig.5 Distribution of PCB congeners detected and content in surficial sediment from Lake Baiyangdian

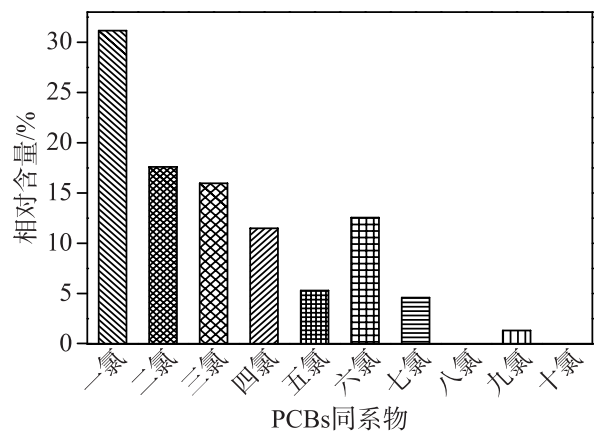

图 6 白洋淀表层沉积物中 PCBs 同系物百分比组成

Fig. 6 Percentage composition of PCBs homologues in surficial sediment from Lake Baiyangdian 
物中 PCBs 分布情况的调查结果 ${ }^{[36]}$, 一氯、二氯联苯等低氯联苯含量明显增多而高氯联苯五氯、六氯联苯含 量明显减少, 这一结果表明白洋淀沉积物中可能存在微生物脱氯现象. 结合国内 PCBs 商品生产状况, 五氯、 六氯联苯含量的大幅度减少以及五氯联苯较低的相对含量在一定程度上可以说明白洋淀地区 PCBs 污染情 况好转且无新的污染源输人.

国内外部分研究地区沉积物中 PCBs 污染情况见表 3. 国外发达国家水体沉积物中 PCBs 含量普遍较 高, 白洋淀 PCBs 含量远低于美国历史上 PCBs 污染严重的哈德逊河和格拉斯河, 与国外研究地区相比, 白洋 淀 PCBs 含量也处于低等水平. 与国内其他地区进行比较, 白洋淀 PCBs 含量较太湖、黄海、滇池等地高, 与珠 江三角洲流域、长江口及东海近岸和太湖竺山湾及人湖河流等处相比 PCBs 含量较低. 总体来说, 白洋淀沉 积物中 PCBs 含量在全国范围内处于中等水平, PCBs 含量与 2011 年白洋淀调查时无明显差异, 但 PCB 单体 种类减少.

表 3 国内外不同地区表层沉积物中 PCBs 含量比较

Tab.3 Comparison of PCBs content in surficial sediments from different areas

\begin{tabular}{|c|c|c|c|c|}
\hline 沉积物 & 年份 & \multicolumn{2}{|c|}{ 检出 PCBs 单体个数 $\Sigma P C B /(n g / g(D W))$} & \multirow{2}{*}{$\frac{\text { 参考文献 }}{[37]}$} \\
\hline 洪湖 & 2019 & 7 & $9.40-22.69$ & \\
\hline 孟加拉国沿海地区 & 2019 & - & $4.61 \sim 105.3$ & {$[38]$} \\
\hline San Diego Bay( 美国加州南部) & 2018 & 24 & $22.5 \sim 1387$ & [39] \\
\hline 巢湖 & 2017 & 41 & $0.03 \sim 24.11$ & {$[40]$} \\
\hline River Ravi and its northern tributaries( 巴基斯坦) & 2017 & 35 & $1.06 \sim 95.76$ & {$[41]$} \\
\hline 白洋淀 & 2017 & 24 & nd $~ 37.61$ & 本研究 \\
\hline 滴水湖 & 2016 & 14 & $0.65 \sim 6.41$ & {$[42]$} \\
\hline 哈德逊河 & 2016 & 145 & 1590 & {$[43]$} \\
\hline Jobos Bay (波多黎各) & 2016 & - & $0.42 \sim 1232$ & {$[44]$} \\
\hline 黄海 & 2013 & 24 & $0.099 \sim 3.13$ & {$[45]$} \\
\hline 嫩江 & 2012 & 29 & $0.83 \sim 4.44$ & {$[46]$} \\
\hline 滇池 & 2011 & 6 & $0.6 \sim 2.4$ & {$[47]$} \\
\hline 白洋淀( 2011 年) & 2011 & 38 & $5.96 \sim 29.61$ & {$[36]$} \\
\hline 全太湖 & 2009 & 56 & $1.35 \sim 13.8$ & {$[48]$} \\
\hline 海河河口 & 2009 & 12 & 25.24 & {$[49]$} \\
\hline 珠江三角洲流域 & 2009 & 128 & $10.16 \sim 485.45$ & {$[50]$} \\
\hline Houston channel(美国) & 2008 & 18 & $0.5 \sim 1418.0$ & {$[51]$} \\
\hline 南四湖 & 2007 & 11 & $7.84 \sim 42.8$ & {$[30]$} \\
\hline 长江三角洲 & 2006 & 23 & $0.92 \sim 9.69$ & {$[52]$} \\
\hline 红枫湖 & 2006 & 7 & $3.2 \sim 31.6$ & {$[32]$} \\
\hline Busan Bay(韩国) & 2005 & 22 & $5.7 \sim 199.0$ & {$[53]$} \\
\hline 长江口潮滩 & 2003 & 77 & $0.19 \sim 18.95$ & {$[54]$} \\
\hline 北极湖泊 & 1996 & - & $2.4 \sim 39.0$ & {$[55]$} \\
\hline Venice Lagoon(意大利) & 1991 & 7 & $4.05 \sim 239.15$ & {$[56]$} \\
\hline
\end{tabular}

\section{3 白洋淀表层沉积物中 PCBs 风险评估}

因部分 OCPs 缺乏标准值, 白洋淀 10 种检出的 OCPs 中, 仅评估 $\gamma$-HCH、Dieldrin 和 p, p ${ }^{\prime}$-DDD, 其余 OCPs 暂不进行风险评估, 结果如表 4 所示.

对于 $\mathrm{p}, \mathrm{p}^{\prime}-\mathrm{DDD}$, 仅有两处采样点含量位于 $E R L$ 值与 $E R M$ 值之间, 其余均低于 $E R L$ 值, 表明白洋淀表层 沉积物中 $\mathrm{p}, \mathrm{p}^{\prime}-\mathrm{DDD}$ 生态风险较低, 只杨家淀、大观园两处存在一定毒性风险; 对于 Dieldrin, 烧车淀湿地采 样点处含量高于 $E R M$, 存在较大可能的生态风险, 同时大部分采样点处的 Dieldrin 含量位于 $E R L$ 值与 $E R M$ 值之间, 均存在一定毒性风险, 尚需进一步关注; 对于 $\gamma-\mathrm{HCH}$, 白洋淀沉积物中 $\gamma$-HCH 生态风险较高, 11 处 采样点中有 7 处采样点的 $\gamma-\mathrm{HCH}$ 含量高于 PEL 值, 不容忽视; 对于 $\sum$ PCBs, 8 处采样点的总 PCBs 含量低于 
$E R L$ 值, 3 处采样点含量介于 $E R L$ 值与 $E R M$ 值之间, 生态风险较低. 整体 $m E R M q$ 值为 1.37 , 大于 0.5 而小于 1.5 , 表明白洋淀研究区域的总体生态风险程度为中, 需要重视.

表 4 白洋淀表层沉积物中 OCPs 和 PCBs 生态风险评估

Tab.4 Ecological risk of OCPs and PCBs in surficial sediments from Lake Baiyangdian

\begin{tabular}{|c|c|c|c|c|c|c|}
\hline \multirow{2}{*}{ 化合物 } & \multicolumn{2}{|c|}{ SQGs } & \multirow{2}{*}{$\begin{array}{c}\text { 含量/ } \\
(\mathrm{ng} / \mathrm{g})\end{array}$} & \multicolumn{3}{|c|}{ 比例 (点位数) } \\
\hline & $E R L$ & $E R M$ & & $<E R L$ & $E R L \sim E R M$ & $>E R M$ \\
\hline $\mathrm{p}, \mathrm{p}^{\prime}-\mathrm{DDD}$ & 2.0 & 20.0 & nd $\sim 2.66$ & $81.8 \%(9)$ & $18.2 \%(2)$ & \\
\hline Dieldrin & 0.02 & 8 & nd $\sim 19.83$ & $18.2 \%(2)$ & $72.7 \%(8)$ & $9.1 \%(1)$ \\
\hline$\gamma$-HCH & $0.32 *$ & $0.99^{*}$ & nd $\sim 20.77$ & $36.4 \%(4)$ & & $63.6 \%(7)$ \\
\hline$\sum$ PCBs & 22.7 & 180 & nd-37.61 & $72.7 \%(8)$ & $27.3 \%(3)$ & \\
\hline
\end{tabular}

* 代表 TEL、PEL 值.

\section{3 结论}

1) 白洋淀表层沉积物中共检出 10 种 OCPs, 总含量在 $1.22 \sim 52.45 \mathrm{ng} / \mathrm{g}$ 之间, 在国内处中等水平, $\mathrm{HCHs}$ 和 Dieldrin 为其主要污染物. HCHs 组成分析发现白洋淀地区可能存在新的 HCHs 污染源输人.

2) 白洋淀表层沉积物中共检出 24 种 PCB 单体, 总含量在 nd 37.61 ng/g 之间, 较国外地区污染程度 低, 在国内处中等水平. 目前 PCBs 分布以低氯联苯为主, 沉积物中低氯联苯含量大幅度上升而高氯联苯含 量大幅度下降, 白洋淀地区 PCBs 污染程度减轻, 无新的污染源输人且可能存在微生物脱氯现象.

3 ) 白洋淀沉积物中, $p, p^{\prime}-$ DDD 和 $\Sigma$ PCBs 生态风险较低,仅个别地区存在一定毒性风险需关注; Dieldrin 尚需关注; $\gamma$ - HCH 在多数地区含量均高于 $P E L$ 值, 存在较高的生态风险.

\section{4 附录}

附录 I IV 见电子版( DOI: 10.18307/2020.0306).

\section{5 参考文献}

[ 1 ] Lyall K, Croen LA, Sjödin A et al. Polychlorinated biphenyl and organochlorine pesticide concentrations in maternal midpregnancy serum samples: Association with autism spectrum disorder and intellectual disability. Environmental Health Perspectives, 2017, 125(3) : 474-480. DOI: 10.1289/ehp277.

[ 2 ] Xu P, Feng YP, Fan J et al. Organochlorine pesticides pollution in soils of typical areas in China: Recent advances and future prospects. Agrochemicals, 2014, 53(3): 164-166. [徐鹏, 封跃鹏, 范洁等. 有机氯农药在我国典型地区土壤 中的污染现状及其研究进展. 农药, 2014, 53(3): 164-166.]

[ 3 ] Fang XH, Qiu RL. Behavior of pesticide in soil environment. Soil and Environment Sciences, 2002, 11(1): 94-97. [ 方晓 航, 仇荣亮. 农药在土壤环境中的行为研究. 土壤与环境, 2002, 11(1):94-97.]

[ 4 ] Chen Y. The status of electric equipment containing PCBs and the environmental management measures in Jiangsu Province. Pollution Control Technology, 2012, 25(3) : 29-30. [陈宇. 江苏省含多氯联苯电力设备现状与环境管理对策. 污染防治技术, 2012, 25(3): 29-30.]

[ 5 ] Yang B, Zhou LL, Xue ND et al. China action of “Cleanup Plan for Polychlorinated Biphenyls Burial Sites”: Emissions during excavation and thermal desorption of a capacitor-burial site. Ecotoxicology and Environmental Safety, 2013, 96: 231-237. DOI: 10.1016/j. ecoenv.2013.06.026.

[ 6 ] Zhang JW, Zhang H, Liu Y et al. Residues characters and health risk assessment of PCBs in agricultural soils of Taiyuan City. Journal of Anhui Agricultural Sciences, 2017, (35): 96-101. [张婧雯, 张红, 刘勇等. 太原市农田土壤中多氯联 苯污染特征及健康风险. 安徽农业科学, 2017, (35) : 96-101.]

[ 7 ] Ding HX, Tao XM, Li DD. Pollution characteristics of organism in dustfall of Lanzhou city. Environmental Study and Monitoring, 2018, (1) : 1-4. [丁海霞, 陶雪梅, 李丹丹. 兰州市大气降尘中有机物的污染特征. 环境研究与监测, 
2018, (1): 1-4.]

[ 8 ] Zhou ZF, Feng SJ, Xie CX et al. Pollution characteristics and distribution in gas-particle phase of polychlorinated biphenyls (PCBs) in the West Third Ring area in Beijing, China. Environmental Chemsitry, 2018, (4) : 871-879. [周仲凡, 冯思捷, 解春宵等. 北京市西三环地区大气中多氯联苯的污染特征和气粒分配. 环境化学, 2018, (4) : 871-879.]

[ 9 ] Xiong L, Han WH, Dang JH et al. Pollution level and characteristics of polychlorinated biphenyls in the Luanhe River Basin. Xiamen: 2017 annual meeting of science and technology of Chinese society of Environmental Sciences, 2017. [ 熊莲, 韩文辉, 党晋华等. 汾河流域多氯联苯污染水平及特征. 厦门: 2017 中国环境科学学会科学与技术年会, 2017.]

[10] Hu GC, Luo XJ, Li FC et al. Organochlorine compounds and polycyclic aromatic hydrocarbons in surface sediment from Baiyangdian Lake, North China: Concentrations, sources profiles and potential risk. Journal of Environmental Sciences, 2010, 22(2) : 176-183. DOI : 10.1016/s1001-0742(09)60090-5.

[11] Da CN, Wu K, Jin J et al. Levels and sources of organochlorine pesticides in surface sediment from anhui reach of Huaihe river, China. Bulletin of Environmental Contamination and Toxicology, 2017, 98(6) : 784-790. DOI: 10.1007/s00128017-2088-4.

[12] Guo W, Zhang HY, Cui SB et al. Assessment of the distribution and risks of organochlorine pesticides in core sediments from areas of different human activity on Lake Baiyangdian, China. Stochastic Environmental Research and Risk Assessment, 2014, 28(4) : 1035-1044. DOI: 10.1007/s00477-013-0799-6.

[13] Guo W, Zhang HY, Huo SL. Organochlorine pesticides in aquatic hydrophyte tissues and surrounding sediments in Baiyangdian wetland, China. Ecological Engineering, 2014, 67: 150-155. DOI: 10.1016/j.ecoleng.2014.03.047.

[14] Dai GH, Liu XH, Liang G et al. Health risk assessment of organochlorine contaminants in fish from a major lake (Baiyangdian lake) in North China. Bulletin of Environmental Contamination and Toxicology, 2011, 87(1) : 58-64. DOI: 10. 1007/s00128-011-0276-1.

[15] Xu L, Liu S, Qing QD et al. Distribution and source apportionment of polychlorinated biphenyl congeners in surface sediments from Zhushan Bay and the inflow rivers of Lake Taihu. China Environmental Science, 2017, 37(11): 4333-4341. [徐否, 刘莎, 秦庆东等. 太湖竺山湾及人湖河流沉积物中多氯联苯单体分布及源解析. 中国环境科学, 2017, 37 (11) : 4333-4341.]

[16] Long ER, MacDonald DD. Recommended uses of empirically derived, sediment quality guidelines for marine and estuarine ecosystems. Human and Ecological Risk Assessment: An International Journal, 1998, 4 ( 5) : 1019-1039. DOI: 10. 1080/10807039891284956.

[17] Hu GC, Dai JY, Mai BX et al. Concentrations and accumulation features of organochlorine pesticides in the Baiyangdian lake freshwater food web of North China. Archives of Environmental Contamination and Toxicology, 2010, 58(3): 700710. DOI: 10.1007/s00244-009-9400-1.

[18] Ding Y, Huang HF, Li H et al. Residues of organochlorine pesticides (OCPs) in water and sediments from Nansha Mangrove Wetland. Environment Science, 2017, 38(4) : 1431-1441. [丁洋, 黄焕芳, 李绘等. 广州南沙红树林湿地水体 和沉积物中有机氯农药的残留特征. 环境科学, 2017, 38(4) : 1431-41.]

[19] Jin XL, Liu Y, Qiao XC et al. Risk assessment of organochlorine pesticides in drinking water source of the Yangtze River. Ecotoxicology and Environmental Safety, 2019, 182: 109390. DOI: 10.1016/j.ecoenv.2019.109390.

[20] Liu J, Ding Y, Qi SH et al. Characteristics of organochlorine pesticides ( OCPs ) in soil samples of Hanjiang River Basin, southeast China. Environment Science, 2018, (11): 1-11. [刘佳, 丁洋, 祁士华等. 韩江流域土壤中有机氯农药的特 征分布. 环境科学, 2018, (11): 1-11.]

[21] Bai Y, Ruan XH, van der Hoek JP. Residues of organochlorine pesticides (OCPs) in aquatic environment and risk assessment along Shaying River, China. Environmental Geochemistry and Health, 2018, 40(6) : 2525-2538. DOI: 10.1007/ s10653-018-0117-9.

[22] Fu X, Liang YP, Qin LT et al. Distribution of organochlorine pesticides( OCPs) in the water body of Huixian Karst wetland of Guilin and environmental risk assessment of OCP mixtures. Journal of Agro-Environment Science, 2018, 37 (5) : 974983. [ 符釒金, 梁延鹏, 覃礼堂等. 桂林会仙岩溶湿地水体中有机氯农药分布特征及混合物环境风险评估. 农业环 境科学学报, 2018, 37(5): 974-983.]

[23] Shen BB, Wu JL, Zhao ZH. Organochlorine pesticides and polycyclic aromatic hydrocarbons in water and sediment of the Bosten Lake, Northwest China. Journal of Arid Land, 2017, 9(2) : 287-298. DOI: 10.1007/s40333-017-0008-4. 
[24] Ding QY, Zhang ZH, Jiang Y et al. Vertical distribution characteristics of organochlorine pesticides and polycyclic aromatic hydrocarbons in a sedimentary core from Zhouxi Bay, Lake Poyang. J Lake Sci, 2016, 28(4) : 765-74. DOI: 10.18307/ 2016.0409. [ 畉倩玉, 赵中华, 蒋豫等. 鄱阳湖周溪湾沉积物中有机氯农药和多环芳烃的垂直分布特征. 湖泊科 学, 2016, 28(4): 765-774.]

[25] Jiang S, Sun BH, Xu B et al. Characteristics and risk assessment of organochlorine pesticides in water and surface sediment from main estuaries of Chaohu Lake. Environmental Chemistry, 2016, 35(6): 1228-1236. [姜珊, 孙丙华, 徐彪 等. 巢湖主要湖口水体和表层沉积物中有机氯农药的残留特征及风险评价. 环境化学, 2016, 35(6): 1228-1236.]

[26] Die QQ, Nie ZQ, Liu F et al. Distribution characteristics of organochlorine pesticides in the soils from the upper reaches of Haihe River. Environmental Science and Technology, 2015, 38(2):83-88. [ 迭庆杞, 聂志强, 刘峰等. 海河上游地区 土壤有氯农药的分布特征研究. 环境科学与技术, 2015, 38(2): 83-88.]

[27] Xu Y, Zhou YZ, Chen X et al. Chemical characterization and risk assessment of organochlorine pesticides in sediments and biota from Meiliang Bay of Taihu Lake. Journal of Southeast University: Natural Science Edition, 2015, 45(2) : 328-335. [许妍, 周亚子, 陈曦等. 太湖梅梁湾沉积物和水生生物中有机氯农药分布特征及风险评估. 东南大学学报: 自然 科学版, 2015, $45(2): 328-335$.]

[28] Chen F, Tang FL, Xu JM et al. Residue characteristics and ecological risk assessment of organochlorine pesticide (OCPs) in the surface sediments of Lake Qiandao. J Lake Sci, 2014, 26(4) : 593-599. DOI: 10.18307/2014.0414. [陈峰, 唐访 良, 徐建芬等. 千岛湖表层沉积物中有机氯农药的残留特征及生态风险评价. 湖泊科学, 2014, 26(4): 593-599.]

[29] Ding J. Temporal and spatial distribution of PCBs, OCPs and PAHs in Songhua River [Dissertation]. Harbin: Harbin Institute of Technology, 2008. [丁晶. 松花江干流 PCBs、OCPs、PAHs 时空分布研究 [学位论文]. 哈尔滨: 哈尔滨工 业大学, 2008.]

[30] Li HL, Li GG, Yang F et al. Vertical distribution characteristics of organochlorine pesticides and polychlorinated biphenyls in sediment core from Lake Nansihu. Environment Science, 2007, (7): 1590-1594. [李红莉, 李国刚, 杨帆等. 南四湖 沉积物中有机氯农药和多氯联苯垂直分布特征. 环境科学, 2007, (7) : 1590-1594.]

[31] Wang YH, Qi SH, Wang W. The characteristic distribution of organic chlorine pesticides in the water and sediment of Diaocha Lake in Hubei Province. Environmental Pollution and Prevention, 2007, (6): 415-418. [王英辉, 祁士华, 王伟. 㲽汉湖水体和表层沉积物中有机氯农药分布特征. 环境污染与防治, 2007, (6): 415-418.]

[32] Wei ZQ. Biogeochemistry of organochlorine pollutants in Hongfeng Reservoir [ Dissertation]. Guiyang: Institute of Geochemistry, Chinese Academy of Sciences, 2006. [魏中青. 红枫湖有机氯污染物的生物地球化学 [学位论文]. 贵阳: 中国科学院地球化学研究所, 2006.]

[33] Gao QS, Jiao LX, Yang L et al. Occurrence and ecological risk assessment of typical persistent organic pollutants in Baiyangdian Lake. Environment Science, 2018, 39(4): 1616-1627. [高秋生, 焦立新, 杨柳等. 白洋淀典型持久性有机污 染物污染特征与风险评估. 环境科学, 2018, 39(4): 1616-1627.]

[34] Xu DD, Zhong WK, Deng LL et al. Regional distribution of organochlorinated pesticides in pine needles and its indication for socioeconomic development. Chemosphere, 2004, 54(6) : 743-752. DOI: 10.1016/j.chemosphere.2003.08.022.

[35] Jiang LF, Wang XD, Sun YZ et al. Residues of organochlorine pesticides in urban soil of Shanghai. Environment Science, 2010, 31(2) : 409-414. [蒋显峰, 王学彤, 孙阳昭等. 上海市城区土壤中有机氯农药残留研究. 环境科学, 2010, 31(2) : 409-414.]

[36] Dai GH, Liu XH, Liang G et al. Distribution of organochlorine pesticides (OCPs) and polychlorinated biphenyls (PCBs) in surface water and sediments from Baiyangdian Lake in North China. Journal of Environmental Sciences, 2011, 23(10): 1640-1649. DOI: 10.1016/s1001-0742(10)60633-x.

[37] Wei LF, Tadesse AW, Wang J. Organohalogenated contaminants (OHCs) in surface sediments and water of East Dongting Lake and Hong Lake, China. Archives of Environmental Contamination and Toxicology, 2019, 76(2) : 157-170. DOI: 10. 1007/s00244-018-0564-4.

[38] Habibullah-Al-mamun M, Ahmed MK, Islam MS et al. Seasonal-spatial distributions, congener profile, and risk assessment of polychlorinated biphenyls (PCBS) in the surficial sediments from the coastal area of Bangladesh. Soil and Sediment Contamination: an International Journal, 2019, 28(1) : 28-50. DOI: 10.1080/15320383.2018.1528575.

[39] Neira C, Vales M, Mendoza G et al. Polychlorinated biphenyls ( PCBs) in recreational marina sediments of San Diego Bay, southern California. Marine Pollution Bulletin, 2018, 126: 204-214. DOI: 10.1016/j.marpolbul.2017.10.096. 
[40] Huo SL, Li CC, Xi BD et al. Historical record of polychlorinated biphenyls ( PCBs) and special occurrence of PCB 209 in a shallow fresh-water lake from Eastern China. Chemosphere, 2017, 184: 832-840. DOI: 10.1016/j. chemosphere.2017. 06.073 .

[41] Baqar M, Sadef Y, Ahmad SR et al. Occurrence, ecological risk assessment, and spatio-temporal variation of polychlorinated biphenyls (PCBs) in water and sediments along River Ravi and its northern tributaries, Pakistan. Environmental Science and Pollution Research, 2017, 24(36) : 27913-27930. DOI: 10.1007/s11356-017-0182-0.

[42] Wang XP, Huang X, Bi CJ et al. Spatial distribution characteristics and risk assessment of polychlorinated biphenyls (PCBs) in sediments and soils from the Dishui Lake and Its river system. Environment Science, 2016, 37 (6) : 21212130. [王薛平, 黄星, 毕春娟等. 滴水湖及其环湖水系沉积物、土壤中多氯联苯的空间分布特征及风险评价. 环 境科学, 2016, 37(6): 2121-2130.]

[43] Xu Y, Gregory KB, VanBriesen JM. Microbial-catalyzed reductive dechlorination of polychlorinated biphenyls in Hudson and Grasse river sediment microcosms: Determination of dechlorination preferences and identification of rare ortho removal pathways. Environmental Science \& Technology, 2016, 50(23): 12767-12778. DOI : 10.1021/acs.est.6b03892.

[44] Alegria H, Martinez-Colon M, Birgul A et al. Historical sediment record and levels of PCBs in sediments and mangroves of Jobos Bay, Puerto Rico. Science of the Total Environment, 2016, 573: 1003-1009. DOI: 10. 1016/j. scitotenv. 2016. 08.165 .

[45] Duan XY, Li YX, Li XG et al. Polychlorinated biphenyls in sediments of the Yellow Sea: Distribution, source identification and flux estimation. Marine Pollution Bulletin, 2013, 76(1/2) : 283-290. DOI: 10.1016/j.marpolbul.2013.08.024.

[46] Nie HF, Zhao LD, Liu YH et al. Distribution, sources and ecological risk assessment of polychlorinated biphenyl in sediments from Songhua River Basin. Environment Science, 2012, 33(10): 3434-3442. [聂海峰, 赵传冬, 刘应汉等. 松花 江流域河流沉积物中多氯联苯的分布、来源及风险评价. 环境科学, 2012, 33(10): 3434-3442.]

[47] Wan X, Pan XJ, Wang B et al. Distributions, historical trends, and source investigation of polychlorinated biphenyls in Dianchi Lake, China. Chemosphere, 2011, 85(3) : 361-367. DOI : 10.1016/j.chemosphere.2011.06.098.

[48] Chen YY, Yin Y, Wang XR et al. Polycyclic aromatic hydrocarbons and polychlorinated biphenyl in surface sediments of Taihu Lake: the distribution, sources and risk assessment. China Environmental Science, 2009, 29(2) : 118-124. [ 陈燕燕, 尹颖, 王晓蓉等. 太湖表层沉积物中 PAHs 和 PCBs 的分布及风险评价. 中国环境科学, 2009, 29(2): 118-124.]

[49] Wang T, Huang J, Yu G et al. Source apportionment of polychlorinated biphenyls and organochlorine pesticides in surface sediments of Haihe Estuary. China Environmental Science, 2009, 29(7) : 722-726. [王泰, 黄俊, 余刚. 海河河口表层 沉积物中 PCBs 和 OCPs 的源解析. 中国环境科学, 2009, 29(7): 722-726.]

[50] Kang YH, Mai BX, Huang XG et al. Primary study on status of organic pollution in surface sediments of the Pearl River Delta. Acta Scientiae Circumstantiae, 2000, (S1) : 164-170. [ 康跃惠, 麦碧涃, 黄秀娥等. 珠江三角洲地区水体表层 沉积物中有机污染状况初步研究. 环境科学学报, 2000, (S1) : 164-170.]

[51] Howell NL, Suarez MP, Rifai HS et al. Concentrations of polychlorinated biphenyls (PCBs) in water, sediment, and aquatic biota in the Houston Ship Channel, Texas. Chemosphere, 2008, 70(4) : 593-606. DOI: 10.1016/j.chemosphere. 2007.07.031.

[52] Shen M, Yu YJ, Zheng GJ et al. Polychlorinated biphenyls and polybrominated diphenyl ethers in surface sediments from the Yangtze River Delta. Marine Pollution Bulletin, 2006, 52(10) : 1299-1304. DOI: 10.1016/j.marpolbul.2006.05.023.

[53] Hong SH, Yim UH, Shim WJ et al. Congener-specific survey for polychlorinated biphenlys in sediments of industrialized bays in Korea: regional characteristics and pollution sources. Environmental Science \& Technology, 2005, 39(19) : 73807388. DOI: $10.1021 / \mathrm{es050397c.}$

[54] Yang Y, Liu M, Xu SY et al. Distribution of polychlorinated biphenyls (PCBs) and organochlorine pesticides (OCPs) in the tidal beach surface sediments of Yangtze estuary. China Environmental Science, 2003, (2): 104-108. [杨毅, 刘敏, 许世远等. 长江口潮滩表层沉积物中 PCBs 和 OCPs 的分布. 中国环境科学, 2003, (2) : 104-108.]

[55] Muir DCG, Omelchenko A, Grift NP et al. Spatial trends and historical deposition of polychlorinated biphenyls in Canadian midlatitude and arctic lake sediments. Environmental Science \& Technology, 1996, 30( 12 ) : 3609-3617. DOI: 10. $1021 /$ es960393v.

[56] Mangani F, Crescentini G, Sisti E et al. PAHs, PCBs and chlorinated pesticides in mediterraneal coastal sediments. International Journal of Environmental Analytical Chemistry, 1991, 45(2) : 89-100. DOI: 10.1080/03067319108026979. 
附录 I OCP 的保留时间与回收率

Appendix I Retention time and recovery of OCPs

\begin{tabular}{|c|c|c|c|}
\hline \multirow{2}{*}{ OCP } & \multirow{2}{*}{ 保留时间/min } & \multicolumn{2}{|c|}{ 回收率/\% } \\
\hline & & 算术平均值 & 标准偏差 \\
\hline$\alpha-\mathrm{HCH}$ & 28.29 & 103.2 & 4.3 \\
\hline$\beta-\mathrm{HCH}$ & 33.83 & 96.0 & 6.1 \\
\hline$\gamma-\mathrm{HCH}$ & 42.00 & 102.0 & 5.1 \\
\hline$\delta-\mathrm{HCH}$ & 45.88 & 95.2 & 3.5 \\
\hline Heptachlor(七氯) & 46.87 & 97.6 & 3.2 \\
\hline Aldrin(艾氏剂) & 53.15 & 93.2 & 7.9 \\
\hline Heptachlor epoxide(七氯环氧化物) & 67.28 & 105.5 & 4.3 \\
\hline trans-Chordane( $\alpha$-氯丹) & 78.77 & 85.3 & 3.7 \\
\hline cis-Chordane( $\beta$-氯丹) & 79.27 & 92.8 & 4.9 \\
\hline Endosulfan I（硫丹 I ） & 79.79 & 85.6 & 5.6 \\
\hline $\mathrm{p}, \mathrm{p}^{\prime}-\mathrm{DDE}$ & 88.48 & 97.6 & 4.1 \\
\hline Dieldrin (狄氏剂) & 91.53 & 88.1 & 3.6 \\
\hline Endrin( 异狄氏剂) & 95.49 & 90.6 & 5.4 \\
\hline Endosulfan II ( 硫丹 II ) & 108.18 & 101.7 & 3.1 \\
\hline $\mathrm{p}, \mathrm{p}^{\prime}-\mathrm{DDD}$ & 111.78 & 105.4 & 8.0 \\
\hline Endrin aldehyde( 异狄氏剂醛) & 112.99 & 95.2 & 2.9 \\
\hline Endosulfan sulfate( 硫丹硫酸盐) & 122.90 & 98.3 & 5.4 \\
\hline $\mathrm{p}, \mathrm{p}^{\prime}-\mathrm{DDT}$ & 125.09 & 101.7 & 4.2 \\
\hline Endrin ketone( 异狄氏剂酮) & 137.75 & 92.7 & 4.7 \\
\hline methoxychlor( 甲氧滴滴涕) & 146.67 & 93.2 & 5.6 \\
\hline
\end{tabular}

附录 II PCB 单体的保留时间与回收率

Appendix II Retention time and recovery of PCBs

\begin{tabular}{|c|c|c|c|c|}
\hline \multirow{2}{*}{ IUPAC\# } & \multirow{2}{*}{ 共析峰 } & \multirow{2}{*}{ 保留时间/min } & \multicolumn{2}{|c|}{ 回收率/\% } \\
\hline & & & 算术平均值 & 标准偏差 \\
\hline 1 & & 16.45 & 86.0 & 2.4 \\
\hline 2 & & 19.94 & 85.6 & 1.5 \\
\hline 3 & & 20.68 & 87.7 & 2.2 \\
\hline 4 & 10 & 21.75 & 85.8 & 0.2 \\
\hline 5 & & 27.39 & 88.4 & 3.6 \\
\hline 6 & & 26.51 & 86.2 & 2.3 \\
\hline 7 & & 25.45 & 87.4 & 3.4 \\
\hline 8 & & 27.79 & 89.9 & 2.0 \\
\hline 9 & & 25.31 & 92.2 & 3.1 \\
\hline 10 & 4 & 21.83 & 85.8 & 0.2 \\
\hline 11 & 18 & 33.99 & 85.3 & 0.3 \\
\hline 12 & & 34.93 & 91.0 & 0.0 \\
\hline 13 & & 35.78 & 90.4 & 1.6 \\
\hline 14 & & 30.40 & 90.4 & 0.6 \\
\hline 15 & & 37.74 & 92.3 & 0.6 \\
\hline 16 & & 37.31 & 87.9 & 2.3 \\
\hline 17 & & 34.59 & 86.9 & 0.3 \\
\hline 18 & 11 & 34.08 & 85.3 & 0.3 \\
\hline
\end{tabular}


续附录 II

\begin{tabular}{|c|c|c|c|c|}
\hline \multirow{2}{*}{ IUPAC\# } & \multirow{2}{*}{ 共析峰 } & \multirow{2}{*}{ 保留时间/min } & \multicolumn{2}{|c|}{ 回收率/\% } \\
\hline & & & 算术平均值 & 标准偏差 \\
\hline 19 & & 29.82 & 92.7 & 2.3 \\
\hline 20 & & 46.67 & 86.0 & 1.7 \\
\hline 21 & & 46.35 & 85.9 & 5.0 \\
\hline 22 & & 49.31 & 87.6 & 1.6 \\
\hline 23 & & 40.39 & 86.4 & 0.8 \\
\hline 24 & & 36.31 & 90.7 & 2.7 \\
\hline 25 & & 43.60 & 89.7 & 2.5 \\
\hline 26 & 50 & 43.05 & 86.3 & 0.3 \\
\hline 27 & & 35.82 & 89.4 & 1.8 \\
\hline 28 & & 46.11 & 89.7 & 0.8 \\
\hline 29 & & 41.15 & 86.0 & 0.9 \\
\hline 30 & & 31.83 & 88.4 & 2.2 \\
\hline 31 & & 45.56 & 88.6 & 1.7 \\
\hline 32 & & 38.12 & 90.6 & 1.1 \\
\hline 33 & & 46.57 & 84.8 & 1.4 \\
\hline 34 & & 40.06 & 87.0 & 0.9 \\
\hline 35 & & 60.22 & 85.7 & 1.8 \\
\hline 36 & 69 & 52.48 & 89.1 & 3.6 \\
\hline 37 & & 63.57 & 87.3 & 1.9 \\
\hline 38 & 75 & 55.816 & 85.6 & 3.2 \\
\hline 39 & $47 \& 62 \& 65$ & 55.61 & 89.0 & 1.6 \\
\hline 40 & 72 & 63.39 & 85.6 & 0.2 \\
\hline 41 & & 61.11 & 78.2 & 1.2 \\
\hline 42 & 59 & 59.36 & 86.0 & 2.2 \\
\hline 43 & & 52.75 & 86.4 & 3.9 \\
\hline 44 & & 58.227 & 87.5 & 4.1 \\
\hline 45 & & 48.76 & 87.9 & 1.6 \\
\hline 46 & & 50.22 & 87.8 & 1.2 \\
\hline 47 & $39 \& 62 \& 65$ & 55.62 & 89.0 & 1.6 \\
\hline 48 & & 54.01 & 85.1 & 3.5 \\
\hline 49 & & 54.63 & 89.8 & 1.8 \\
\hline 50 & 26 & 43.05 & 86.3 & 0.3 \\
\hline 51 & & 47.02 & 85.9 & 1.5 \\
\hline 52 & & 53.50 & 86.9 & 4.5 \\
\hline 53 & & 45.65 & 87.3 & 1.3 \\
\hline 54 & & 39.67 & 90.2 & 1.0 \\
\hline 55 & & 75.79 & 87.1 & 1.8 \\
\hline 56 & & 78.07 & 86.8 & 5.7 \\
\hline 57 & 94 & 66.74 & 88.8 & 1.7 \\
\hline 58 & 67 & 68.20 & 88.4 & 1.0 \\
\hline 59 & 42 & 59.25 & 86.0 & 2.2 \\
\hline 60 & & 79.73 & 85.4 & 4.4 \\
\hline 61 & & 69.04 & 88.5 & 1.8 \\
\hline 62 & $39 \& 47 \& 65$ & 55.69 & 89.0 & 1.6 \\
\hline 63 & & 70.67 & 85.8 & 5.8 \\
\hline
\end{tabular}


续附录 II

\begin{tabular}{|c|c|c|c|c|}
\hline \multirow{2}{*}{ IUPAC\# } & \multirow{2}{*}{ 共析峰 } & \multirow{2}{*}{ 保留时间/min } & \multicolumn{2}{|c|}{ 回收率/\% } \\
\hline & & & 算术平均值 & 标准偏差 \\
\hline 64 & & 63.17 & 92.1 & 2.3 \\
\hline 65 & $39 \& 47 \& 62$ & 55.56 & 89.0 & 1.6 \\
\hline 66 & & 74.07 & 87.3 & 1.3 \\
\hline 67 & 58 & 68.17 & 88.4 & 1.0 \\
\hline 68 & & 64.57 & 90.5 & 2.3 \\
\hline 69 & 36 & 52.59 & 89.1 & 3.6 \\
\hline 70 & & 72.96 & 93.0 & 1.9 \\
\hline 71 & & 60.82 & 87.8 & 3.8 \\
\hline 72 & 40 & 63.47 & 85.6 & 0.2 \\
\hline 73 & & 51.78 & 85.5 & 2.9 \\
\hline 74 & & 71.94 & 87.9 & 0.0 \\
\hline 75 & 38 & 56.139 & 85.6 & 3.2 \\
\hline 76 & 93 & 70.32 & 86.8 & 4.2 \\
\hline 77 & & 97.71 & 86.5 & 0.0 \\
\hline 78 & & 89.33 & 90.6 & 1.1 \\
\hline 79 & & 87.10 & 88.0 & 0.9 \\
\hline 80 & & 77.00 & 87.8 & 3.7 \\
\hline 81 & & 93.67 & 87.8 & 3.6 \\
\hline 82 & & 95.86 & 87.2 & 5.5 \\
\hline 83 & 119 & 84.67 & 88.8 & 1.4 \\
\hline 84 & 89 & 77.79 & 90.1 & 0.0 \\
\hline 85 & & 91.15 & 93.3 & 0.5 \\
\hline 86 & 112 & 85.08 & 87.7 & 1.8 \\
\hline 87 & 136 & 89.62 & 89.0 & 5.7 \\
\hline 88 & & 71.30 & 86.6 & 1.7 \\
\hline 89 & 84 & 77.83 & 90.1 & 0.0 \\
\hline 90 & & 80.31 & 86.7 & 0.6 \\
\hline 91 & & 73.78 & 89.9 & 2.2 \\
\hline 92 & & 78.50 & 87.7 & 2.0 \\
\hline 93 & 76 & 70.32 & 86.8 & 4.2 \\
\hline 94 & 57 & 66.69 & 88.8 & 1.7 \\
\hline 95 & & 71.42 & 90.4 & 0.0 \\
\hline 96 & & 61.71 & 89.6 & 3.1 \\
\hline 97 & & 86.39 & 90.0 & 0.7 \\
\hline 98 & & 69.93 & 89.0 & 0.2 \\
\hline 99 & & 82.08 & 85.7 & 2.0 \\
\hline 100 & & 66.23 & 92.0 & 4.5 \\
\hline 101 & & 80.54 & 94.9 & 2.0 \\
\hline 102 & & 68.95 & 91.2 & 0.1 \\
\hline 103 & & 64.26 & 92.0 & 0.7 \\
\hline 104 & & 55.29 & 92.1 & 4.2 \\
\hline 105 & & 115.14 & 89.6 & 1.3 \\
\hline 106 & 142 & 104.11 & 86.2 & 1.7 \\
\hline 107 & & 103.19 & 86.3 & 2.3 \\
\hline 108 & 125 & 85.75 & 88.0 & 3.1 \\
\hline
\end{tabular}


续附录 II

\begin{tabular}{|c|c|c|c|c|}
\hline \multirow{2}{*}{ IUPAC\# } & \multirow{2}{*}{ 共析峰 } & \multirow{2}{*}{ 保留时间/min } & \multicolumn{2}{|c|}{ 回收率/\% } \\
\hline & & & 算术平均值 & 标准偏差 \\
\hline 109 & 134 & 103.96 & 88.3 & 0.3 \\
\hline 110 & & 93.14 & 89.0 & 6.7 \\
\hline 111 & & 92.13 & 90.1 & 0.9 \\
\hline 112 & 86 & 85.08 & 87.7 & 1.8 \\
\hline 113 & & 81.09 & 90.6 & 0.4 \\
\hline 114 & & 109.00 & 93.2 & 4.6 \\
\hline 115 & & 90.76 & 86.1 & 5.6 \\
\hline 116 & & 87.56 & 87.4 & 6.2 \\
\hline 117 & & 90.49 & 93.0 & 1.4 \\
\hline 118 & & 105.95 & 87.3 & 3.1 \\
\hline 119 & 83 & 84.67 & 88.8 & 1.4 \\
\hline 120 & & 94.30 & 87.2 & 3.2 \\
\hline 121 & & 73.32 & 85.9 & 4.7 \\
\hline 122 & 184 & 107.95 & 89.1 & 3.7 \\
\hline 123 & & 103.68 & 88.3 & 3.4 \\
\hline 124 & 140 & 102.19 & 87.9 & 0.1 \\
\hline 125 & 108 & 85.48 & 88.0 & 3.1 \\
\hline 126 & & 131.35 & 91.0 & 6.2 \\
\hline 127 & & 119.37 & 90.0 & 1.4 \\
\hline 128 & 159 & 132.92 & 93.1 & 4.6 \\
\hline 129 & 163 & 123.15 & 91.8 & 0.1 \\
\hline 130 & & 120.27 & 91.6 & 2.4 \\
\hline 131 & 188 & 105.50 & 88.8 & 2.9 \\
\hline 132 & 161 & 111.08 & 91.7 & 3.1 \\
\hline 133 & & 107.30 & 87.2 & 3.5 \\
\hline 134 & 109 & 104.08 & 88.3 & 0.3 \\
\hline 135 & & 97.47 & 92.3 & 0.0 \\
\hline 136 & 87 & 89.70 & 89.0 & 5.7 \\
\hline 137 & & 118.49 & 92.2 & 4.8 \\
\hline 138 & 160 & 122.50 & 88.3 & 5.3 \\
\hline 139 & 143 & 101.21 & 88.6 & 1.5 \\
\hline 140 & & 102.11 & 87.9 & 0.1 \\
\hline 141 & 176 & 116.38 & 90.0 & 0.5 \\
\hline 142 & 106 & 104.43 & 86.2 & 1.7 \\
\hline 143 & 139 & 101.16 & 88.6 & 1.5 \\
\hline 144 & & 98.57 & 89.8 & 2.4 \\
\hline 145 & & 85.90 & 92.6 & 2.6 \\
\hline 146 & & 109.87 & 89.8 & 2.7 \\
\hline 147 & & 99.87 & 89.1 & 1.0 \\
\hline 148 & & 89.33 & 88.8 & 2.8 \\
\hline 149 & & 100.42 & 89.7 & 5.7 \\
\hline 150 & & 82.01 & 88.6 & 0.5 \\
\hline 151 & & 96.76 & 86.9 & 0.0 \\
\hline 152 & & 83.37 & 85.8 & 1.6 \\
\hline 153 & & 112.51 & 93.4 & 0.9 \\
\hline
\end{tabular}


续附录 II

\begin{tabular}{|c|c|c|c|c|}
\hline \multirow{2}{*}{ IUPAC\# } & \multirow{2}{*}{ 共析峰 } & \multirow{2}{*}{ 保留时间/min } & \multicolumn{2}{|c|}{ 回收率/\% } \\
\hline & & & 算术平均值 & 标准偏差 \\
\hline 154 & & 92.01 & 89.0 & 1.3 \\
\hline 155 & & 74.42 & 90.3 & 2.2 \\
\hline 156 & & 146.11 & 92.1 & 5.3 \\
\hline 157 & 172 & 147.46 & 93.7 & 0.2 \\
\hline 158 & 178 & 124.35 & 86.4 & 3.8 \\
\hline 159 & 128 & 133.02 & 93.1 & 4.6 \\
\hline 160 & 138 & 122.68 & 88.3 & 5.3 \\
\hline 161 & 132 & 110.85 & 91.7 & 3.1 \\
\hline 162 & & 135.12 & 92.6 & 2.2 \\
\hline 163 & 129 & 123.32 & 91.8 & 0.1 \\
\hline 164 & & 121.06 & 94.7 & 2.8 \\
\hline 165 & & 109.36 & 86.9 & 1.6 \\
\hline 166 & & 128.70 & 92.4 & 8.6 \\
\hline 167 & 202 & 137.79 & 98.3 & 3.9 \\
\hline 168 & & 112.22 & 92.4 & 2.6 \\
\hline 169 & 203 & 165.38 & 89.5 & 2.2 \\
\hline 170 & & 161.23 & 94.1 & 0.3 \\
\hline 171 & $201 \& 204$ & 141.62 & 95.8 & 11.4 \\
\hline 172 & 157 & 147.45 & 93.7 & 0.2 \\
\hline 173 & & 141.64 & 95.6 & 3.8 \\
\hline 174 & & 135.54 & 94.3 & 1.3 \\
\hline 175 & 182 & 126.50 & 90.7 & 6.1 \\
\hline 176 & 141 & 116.72 & 90.0 & 0.5 \\
\hline 177 & & 139.42 & 90.3 & 6.6 \\
\hline 178 & 158 & 124.27 & 86.4 & 3.8 \\
\hline 179 & & 113.89 & 88.6 & 1.3 \\
\hline 180 & & 150.43 & 101.7 & 0.2 \\
\hline 181 & & 137.23 & 90.1 & 10.6 \\
\hline 182 & 175 & 126.51 & 90.7 & 6.1 \\
\hline 183 & & 129.93 & 90.9 & 5.7 \\
\hline 184 & 122 & 108.21 & 89.1 & 3.7 \\
\hline 185 & & 133.99 & 94.5 & 8.7 \\
\hline 186 & & 118.02 & 93.3 & 1.6 \\
\hline 187 & & 127.69 & 95.0 & 6.4 \\
\hline 188 & 131 & 105.41 & 88.8 & 2.9 \\
\hline 189 & & 178.01 & 99.8 & 0.4 \\
\hline 190 & & 163.32 & 98.4 & 2.9 \\
\hline 191 & & 153.22 & 95.5 & 6.5 \\
\hline 192 & & 148.65 & 91.8 & 4.9 \\
\hline 193 & & 151.34 & 90.5 & 3.9 \\
\hline 194 & & 188.10 & 98.0 & 4.3 \\
\hline 195 & 207 & 177.86 & 95.8 & 6.0 \\
\hline 196 & & 165.60 & 98.5 & 2.4 \\
\hline 197 & & 144.53 & 99.8 & 1.4 \\
\hline 198 & & 162.29 & 94.2 & 0.9 \\
\hline
\end{tabular}


续附录 II

\begin{tabular}{ccccc}
\hline \multirow{2}{*}{ IUPAC\# } & 共析峰 & 保留时间 $/$ min & \multicolumn{2}{c}{ 回收率/\% } \\
\cline { 4 - 5 } & & & 算术平均值 & 标准偏差 \\
\hline 199 & & 163.00 & 98.1 & 3.9 \\
200 & $171 \& 204$ & 150.28 & 96.9 & 3.6 \\
201 & 167 & 138.04 & 95.8 & 11.4 \\
202 & 169 & 166.04 & 98.3 & 3.9 \\
203 & $171 \& 201$ & 141.62 & 89.5 & 2.2 \\
204 & & 190.25 & 95.8 & 2.0 \\
205 & & 196.30 & 94.9 & 1.3 \\
206 & 195 & 177.86 & 94.9 & 6.0 \\
207 & & 174.28 & 102.6 & 2.6 \\
208 & & 200.85 & 95.9 & 2.0 \\
\hline
\end{tabular}

附录 III 白洋淀沉积物中 OCPs 含量 *

Appendix III Content of OCPs in sediments from Lake Baiyangdian

\begin{tabular}{|c|c|c|c|c|c|c|c|c|c|c|c|c|}
\hline \multirow{2}{*}{ OCPs } & \multicolumn{12}{|c|}{ OCPs 含量/ $(n g / g)$} \\
\hline & BY1 & BY2 & BY3 & BY4 & BY5 & BY6 & BY7 & BY8 & BY9 & BY10 & BY11 & 平均值 \\
\hline$\alpha-\mathrm{HCH}$ & 0.56 & 0.44 & 0.41 & 0.88 & 4.57 & 0.49 & 2.90 & 0.73 & 1.25 & 1.22 & 0.43 & 1.26 \\
\hline$\beta-\mathrm{HCH}$ & 0.00 & 0.00 & 3.09 & 0.00 & 0.00 & 2.03 & 2.77 & 0.00 & 0.00 & 0.00 & 2.18 & 0.92 \\
\hline$\gamma-\mathrm{HCH}$ & 1.57 & 5.30 & 3.00 & 0.00 & 20.77 & 0.00 & 0.00 & 7.14 & 6.99 & 0.00 & 6.38 & 4.65 \\
\hline$\delta-\mathrm{HCH}$ & 0.00 & 0.00 & 0.00 & 0.00 & 0.00 & 0.00 & 0.00 & 0.00 & 0.00 & 0.00 & 2.03 & 0.18 \\
\hline Heptachlor(七氯) & 0.00 & 0.00 & 0.00 & 0.00 & 0.00 & 0.00 & 0.00 & 0.00 & 0.00 & 0.00 & 0.00 & 0.00 \\
\hline Aldrin(艾氏剂) & 0.00 & 0.00 & 0.00 & 0.00 & 0.00 & 0.00 & 0.00 & 1.53 & 5.71 & 0.00 & 13.48 & 1.88 \\
\hline Heptachlor epoxide(七氯环氧化物) & 0.00 & 0.00 & 0.00 & 0.00 & 0.00 & 0.00 & 0.00 & 0.00 & 0.00 & 0.00 & 0.00 & 0.00 \\
\hline trans-Chordane( $\alpha$-氯丹) & 0.00 & 0.00 & 0.00 & 0.00 & 0.00 & 0.00 & 0.00 & 0.00 & 0.00 & 0.00 & 0.00 & 0.00 \\
\hline cis-Chordane( $\beta$-氯丹) & 0.00 & 0.00 & 0.00 & 0.00 & 0.00 & 0.00 & 0.00 & 0.00 & 0.00 & 0.00 & 0.00 & 0.00 \\
\hline Endosulfan I（硫丹 I ） & 0.00 & 0.00 & 0.00 & 0.00 & 0.00 & 0.00 & 0.00 & 0.00 & 0.00 & 0.00 & 0.00 & 0.00 \\
\hline $\mathrm{p}, \mathrm{p}^{\prime}-\mathrm{DDE}$ & 0.00 & 0.00 & 0.00 & 0.00 & 0.00 & 0.00 & 0.00 & 0.00 & 0.00 & 0.00 & 0.00 & 0.00 \\
\hline Dieldrin( 狄氏剂) & 3.34 & 6.00 & 5.80 & 7.96 & 6.35 & 0.00 & 2.21 & 3.64 & 5.80 & 0.00 & 19.83 & 5.54 \\
\hline Endrin( 异狄氏剂) & 0.00 & 0.00 & 0.00 & 0.00 & 0.00 & 0.00 & 0.00 & 0.00 & 0.00 & 0.00 & 0.00 & 0.00 \\
\hline Endosulfan II ( 硫丹 II ) & 0.00 & 0.00 & 0.00 & 0.00 & 0.00 & 0.00 & 0.00 & 0.00 & 0.00 & 0.00 & 0.00 & 0.00 \\
\hline $\mathrm{p}, \mathrm{p}^{\prime}-\mathrm{DDD}$ & 0.00 & 0.00 & 2.24 & 0.00 & 0.00 & 0.00 & 0.00 & 0.00 & 0.00 & 0.00 & 2.66 & 0.45 \\
\hline Endrin aldehyde( 异狄氏剂醛) & 0.00 & 0.00 & 0.00 & 0.00 & 0.00 & 0.00 & 0.00 & 0.00 & 0.00 & 0.00 & 0.00 & 0.00 \\
\hline Endosulfan sulfate( 硫丹硫酸盐) & 0.00 & 0.00 & 0.00 & 0.00 & 0.00 & 0.00 & 0.00 & 0.00 & 0.00 & 0.00 & 5.46 & 0.50 \\
\hline $\mathrm{p}, \mathrm{p}^{\prime}$-DDT & 0.00 & 0.00 & 0.00 & 0.00 & 0.00 & 0.00 & 0.00 & 0.00 & 0.00 & 0.00 & 0.00 & 0.00 \\
\hline Endrin ketone(异狄氏剂酮) & 0.00 & 0.00 & 0.00 & 0.00 & 0.00 & 0.00 & 0.00 & 0.00 & 3.41 & 0.00 & 0.00 & 0.31 \\
\hline methoxychlor(甲氧滴滴涕) & 0.00 & 0.00 & 0.00 & 0.00 & 0.00 & 0.00 & 0.00 & 11.30 & 9.65 & 0.00 & 0.00 & 1.90 \\
\hline$\Sigma O C P$ & 5.46 & 11.74 & 14.54 & 8.84 & 31.70 & 2.52 & 7.88 & 24.33 & 32.81 & 1.22 & 52.45 & \\
\hline
\end{tabular}

$* \mathrm{BY} 1$ 为苲淀, $\mathrm{BY} 2$ 为荷花淀, $\mathrm{BY} 3$ 为杨家淀, $\mathrm{BY} 4$ 为光淀, $\mathrm{BY} 5$ 为鹍巻岛, BY6 为李郎村, $\mathrm{BY} 7$ 为烧车淀河道, $\mathrm{BY} 8$ 为烧车 淀中心, BY9 为郭里口, BY10 为大观园, BY11 为烧车淀湿地. 
附录IV 白洋淀表层沉积物中检出的 PCBs 单体及含量

Appendix IV Distribution of PCB congeners detected and content in surficial sediment from Lake Baiyangdian

\begin{tabular}{|c|c|}
\hline 采样点 & 单体及含量/ $(\mathrm{ng} / \mathrm{g})$ \\
\hline BY1 & PCB30, 2.51 \\
\hline BY2 & РCB30, 1.94 \\
\hline BY3 & PCB1,5.50; РCB 18, 7.47; РCB25, 1.74;РCB29, 1.21; РCB52, 4.81; РCB64,0.94; PCB147, 7.57 \\
\hline BY4 & PCB8, 2.36 \\
\hline BY5 & PCB8, 12.28; РCB96, 8.39;PCB159,3.64 \\
\hline \multicolumn{2}{|l|}{ BY6 } \\
\hline BY7 & PCB1,4.76; РCB30,0.98;PCB74,1.46;PCB81,0.65;PCB117,0.31; РCB147,1.06 \\
\hline BY8 & PCB1,4.67;PCB8,2.52;PCB29,1.18;РCB30,0.89; PCB52,0.98; РCB74,0.65;PCB96,1.16; PCB147, 0.73 \\
\hline BY9 & PCB1 ,6.53;PCB30,1.29; PCB63,2.42;PCB147, 2.90;PCB152,3.20; PCB178,0.70 \\
\hline BY10 & PCB $1,7,45 ;$ PCB206,3.51 \\
\hline BY11 & $\begin{array}{l}\text { PCB1 4.61; PCB8, } 5.22 \text {;PCB29,1.81; PCB30, 2.40; PCB52,2.68; PCB53,3.42; PCB64, 1.08; PCB132, } 1.61 ; \\
\text { PCB147,3.25;PCB160,1.85;PCB188,2.16; PCB178,5.44;PCB181 ,2.06 }\end{array}$ \\
\hline
\end{tabular}

